\title{
Finite Element Rolling Simulation of Pure Copper Plates in Voronoi Polycrystalline Model
}

\author{
Ya-Jie HUANG ${ }^{1, a}$, Yong-Jin $\mathrm{CHEN}^{1, b^{*}}$, Liang MAO ${ }^{1, \mathrm{c}}$ and Yi-Hua ZHENG ${ }^{1, \mathrm{~d}}$ \\ ${ }^{1}$ Department of Engineering Mechanics, Kunming University of Science and Technology, \\ Kunming650500,China
}

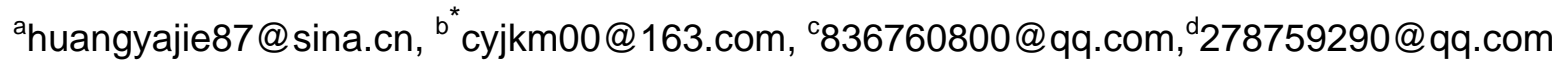

Keywords: Voronoi diagram; polycrystalline; Python scripting interface; mesoscopic.

\begin{abstract}
Generally, in the mesoscopic simulation of polycrystalline materials, correctly establishing polycrystalline models is the key to numerical simulate rolling process. Based on the principle of Voronoi diagram, using MATLAB and ABAQUS Python script interface, the rolling process of pure copper plates in the Voronoi polycrystalline meso-structure model was finite element simulated on ABAQUS platform in this paper. The result shows that because internal each grain has different orientations and interactions with each other, polycrystalline plates display an uneven deformation field in the rolling process and there is obvious stress mutation at grain boundary. These are consistent with the relevant experimental research results and reasonably explain deformation process of polycrystalline materials in theory.
\end{abstract}

\section{Introduction}

Metal materials are mostly a collection of grains, namely polycrystal. Generally, in engineering, the assumption that materials are continuous, homogeneous and isotropic based on the statistical significance is the premise of mechanical analysis of polycrystalline material. This assumption, or constitutive relation, was usually established by macro experiments. Therefore, usually the constitutive relation can be able to meet the needs of strength design and application of engineering. However, when it comes to questions such as large deformation, plastic deformation, fatigue damage and destruction associated with the mesoscopic [1] or microscopic mechanism of material, the analysis on mesoscopic scale becomes necessary. Therefore, research to the evolution of deformation of the internal grains in the process of metal forming process on mesoscopic scale will be of important theoretical significance and engineering practice value.

For forming process of metal materials, the finite element method combined with polycrystalline mesoscopic model is an effective method to predict plastic deformation law for polycrystalline material. Especially, the fact that how to introduce mesoscopic models of polycrystalline material into finite element method has always been a difficult problem and no unified and effective method is found.

\section{Polycrystalline material modeling by Voronoi diagram on mesoscopic scale}

Generally, establishing methods for polycrystalline models are all using Voronoi diagram method. The polycrystalline model established by adopting Voronoi diagram method is called Voronoi polycrystalline model.

At present, domestic scholars have done a lot of work in this aspect. For example, Liangying Si [2] and Huaihui Ren [3] etc have done some work in 2D modeling aspect, while in 3D modeling aspect, Fengguo Zhang [4] etc have some achievements. However, at present, establishing methods of domestic models exist deficiencies, such as complexity, besides, it will take too much time to master so many programming languages. These issues can be improved by using MPT toolbox of MATLAB.

1.1 The definition and generating principle of Voronoi diagram 
2D Voronoi diagram is generally defined as follows: Suppose $\mathrm{S}$ is a set containing n points which are different from each other on the plane, $S=\left\{p_{1}, p_{2}, \ldots \ldots p_{n}\right\}$, as belows:

$$
V\left(p_{i}\right)=\cap\left\{p \mid d\left(p, p_{i}\right)<d\left(p, p_{j}\right)\right\} \quad(\mathrm{i} \neq \mathrm{j}, \mathrm{i}, \mathrm{j}=1,2, \ldots \mathrm{n})
$$

From the above formula, $d\left(p, p_{i}\right)$ express the Euclidean distance between point $p$ and point $p_{i}$, and then this $V\left(p_{i}\right)$ is called Voronoi polygon of the point $p_{i}$. A Voronoi polygon represents a grain of the polycrystal.

\subsection{Python scripting interface in ABAQUS}

Python [5] is a powerful descriptive programming language with efficient data structure, which can be able to achieve object-oriented programming simply and effectively. Most of large engineering application analysis software platform provide an interface for Python for its concise and easy grammar, the special support for dynamic input and the nature of interpreted language. For instance, the ABAQUS scripting interface [6] can customize development on the basis of Python language by extending object model and data types of the Python, thus making function of the ABAQUS scripting interface more powerful. Generally, the ABAQUS scripting interface is mainly used for preprocessing (for example, rapid modeling), post-processing (for example, creating and accessing the database), custom module (for example, automatic post-processing), etc.

1.3 modeling method for Polycrystalline models in ABAQUS

The internal structure of polycrystalline material is very complex, however, Voronoi diagram can better reflect this structural characteristic. Each Voronoi polygon can represent a grain in polycrystalline mesoscopic structure. This paper achieves establishing Voronoi polycrystalline model on the platform of ABAQUS by using MATLAB and Python combined programming.

In MATLAB programming phase, geometry information of points contained by each grain in Voronoi diagram can be obtained through MPT toolbox. Then find the intersection of these information and construct complete topology information of each grain. Finally, output the data information which has been constructed before into a txt file according to certain order so that subsequent Python script can call these data conveniently.

In Python programming phase, we will call the data generated from MATLAB by Python scripting interface of ABAQUS for modeling. Modeling process is as follows:

(1) Read information of each point in each grain into ABAQUS from the txt file generated by the previous step. Store these information in a multilevel list according to the structure by dot, line, surface. Then generate edges of each grain by WirePloyLine command. Finally generate the whole grain using CoverEdges command.

(2) In Assembly module, all grains are eventually merged into a complete Voronoi polycrystalline model by Merge command. Figure 1 is the finite element polycrystalline model containing 100 grains.

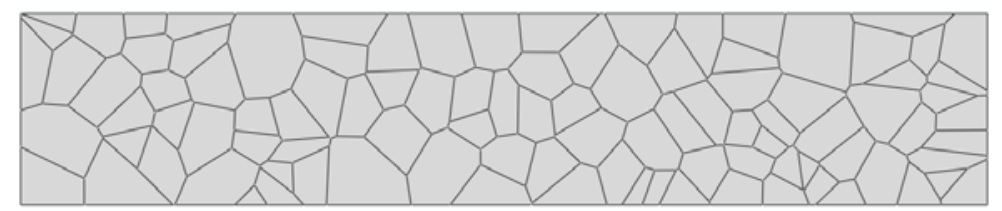

Fig.1 Polycrystalline model containing 100 grains

\section{4 automatic preprocessing based on Python scripting in ABAQUS}

Due to the structure of polycrystalline material mesoscopic model is very complex, it is required that people repeatedly input a large number of parameters and set up multiple dialogs if choosing manual preprocessing. If changing certain parameter of polycrystalline model, the whole preprocessing needs to be dealt again, meanwhile, spending much precious time. Therefore, this paper writes Python scripting module with which rapid preprocessing for different polycrystalline models can be achieved. Script encapsulates grid division, material attributes given, boundary and loading conditions and solving calculation command, etc. Users only need to input the necessary parameters, and then ABAQUS/CAE will automatically preprocess and submit the job to finite element analysis. Automatic preprocessing program flow chart is as below Figure 2. 


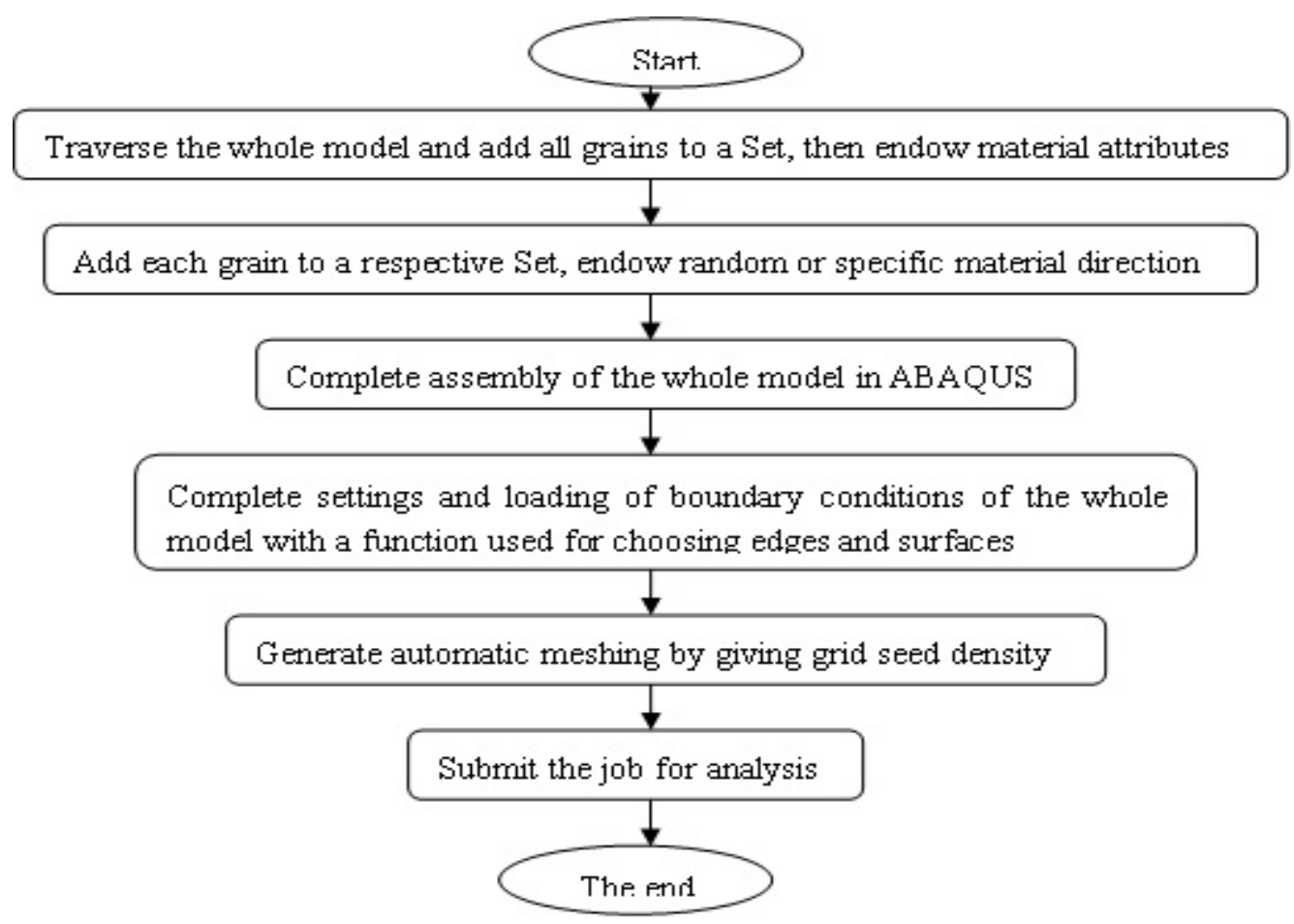

Fig.2 Automatic preprocessor flow diagram of polycrystalline model

\section{Polycrystalline rolling numerical simulation}

In order to probe into the internal deformation process of polycrystalline material in rolling process, firstly this paper conduct finite element rolling simulation for pure copper plate on mesoscopic scale in polycrystalline model, which is anisotropic and has different orientation for each grain. Secondly, similarly conduct finite element rolling simulation for pure copper plate on macroscale in homogeneous, continuous and isotropic model. At last, contrast the respective simulation results of the two models for analysis. Particularly for rolling simulation of polycrystalline model, here random texture is used as the initial texture for finite element rolling simulation of pure copper plate in polycrystalline model.

2.1 Polycrystalline rolling finite element simulation

Under plane strain condition, adoptive model sample is a rectangle of $10 \mathrm{~mm}$ long and $2 \mathrm{~mm}$ high, containing 100 grains, among which each grain orientation is generated by random algorithm. Grid density in each grain is $0.06 \mathrm{~mm}$. The entire model sample has 6292 units, of which including 6128 CPE4R units and 164 CPE3 units. Roller is a rigid body of $40 \mathrm{~mm}$ diameter. Due to the convergent difficulties of rolling calculation, roll down $0.2 \mathrm{~mm}$ before rolling so as to establish effective contact, and then start rolling process at a rotational speed of $2 \mathrm{r} / \mathrm{min}$ after the rolling down step completed. In rolling down process, fix the displacement at the bottom of the model in vertical direction and the tail of the model in horizontal direction. During rolling process, only displacement at the bottom of the model was fixed in vertical direction.

Material parameters of the model are all taken from reference [7], the elastic constants of single crystal materials are as follows: $C_{11}=51227 \mathrm{Mpa}, C_{12}=36930 \mathrm{Mpa}, C_{44}=22937 \mathrm{Mpa}$.

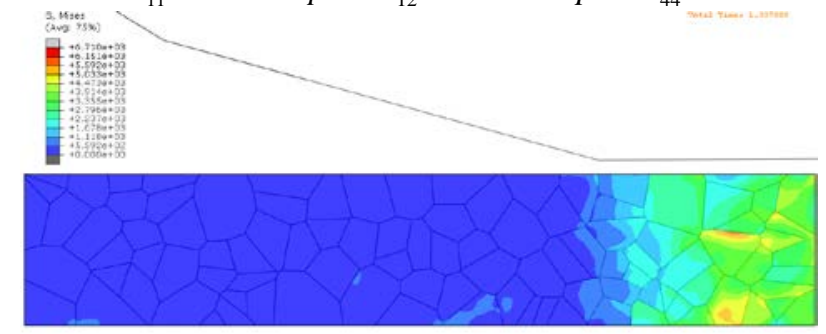

(a) Mises stress distribution when rolling process begin 


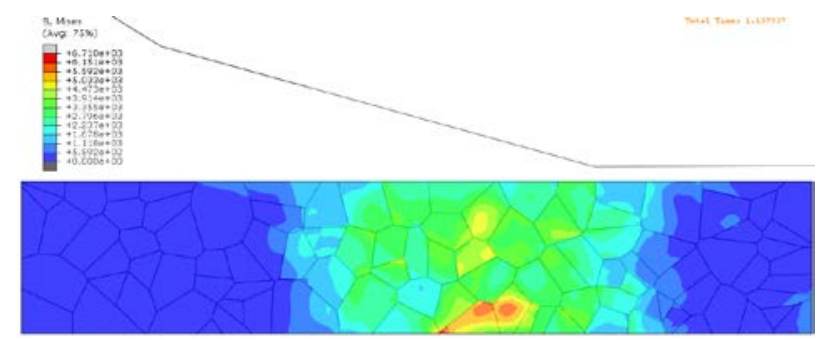

(b) Mises stress distribution in the middle moment of rolling process

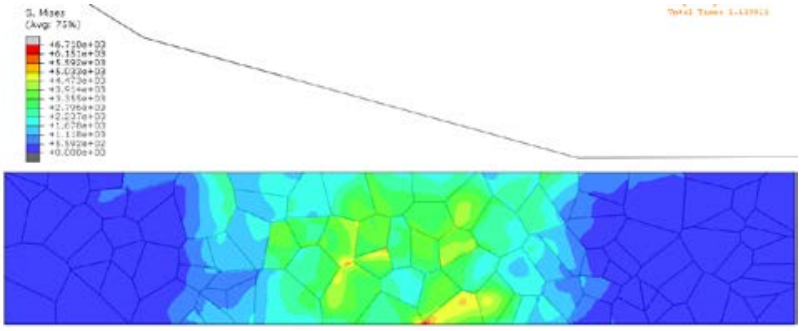

(c) Mises stress distribution when rolling process is about to end

Fig.3 Simulation results of polycrystalline model

2.2 Rolling finite element simulation on macroscale

In order to study the differences of material mechanics performance on different scales, in contrast, this paper also conduct rolling finite element analysis for pure copper plate in homogeneous and isotropic model only considering statistical significance of materials on macroscale.

Keep the size of model sample invariant as a rectangle of $10 \mathrm{~mm}$ long and $2 \mathrm{~mm}$ high. Directly go into Mesh module and then divide the entire model sample into a total of 6286 units. It is obviously seen that the grid density of the macroscopic model is almost the same with the above of the polycrystalline model. Afterwards, boundary and loading conditions remain the same as the step in polycrystalline model.

Material parameters of the macroscopic model are set as follows: $E=43885 \mathrm{Mpa}, v=0.3$.

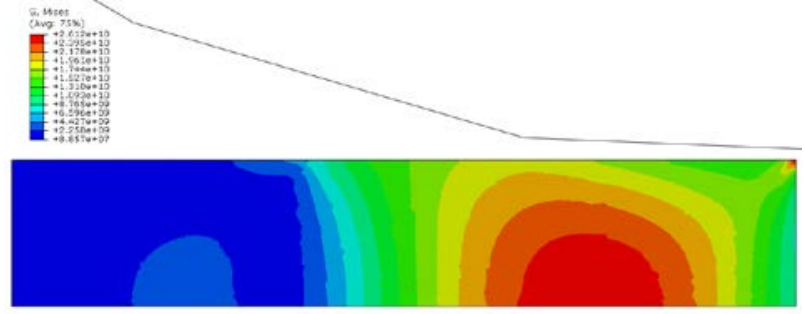

(a) Mises stress contour diagram when rolling process begin

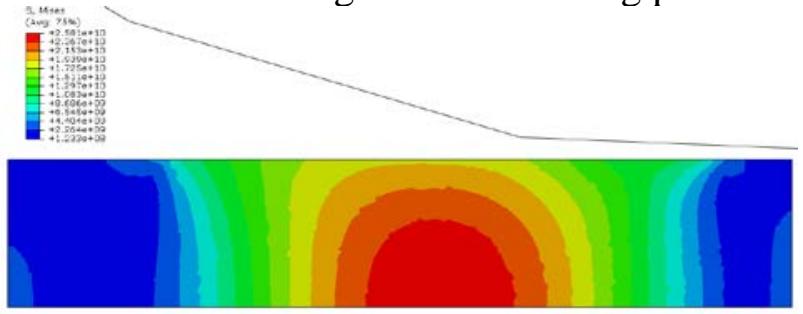

(b) Mises stress contour diagram in the middle moment of rolling process

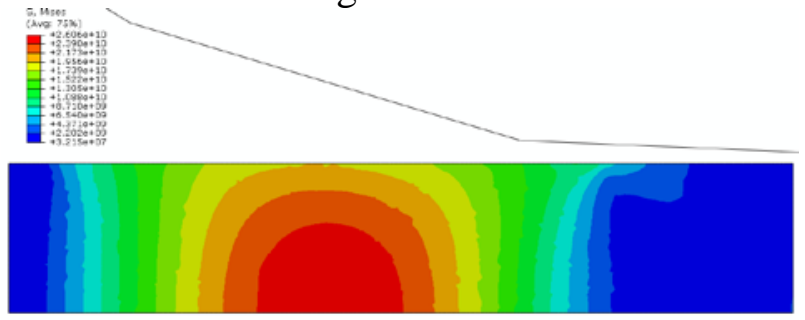

(c) Mises stress contour diagram when rolling process is about to end Fig.4 Simulation results of uniform and isotropic model

2.3 Analysis and discussion of simulation results 
As the graphic result shows: on mesoscopic scale, in fact, each grain has different orientation and materials show anisotropy, in addition, different grains exsit interactions with each other, which result in uneven status of the stress and strain inside polycrystalline model in rolling process, as shown in figure 3 (a), (b) and (c). In some places plastic deformation occurs first and it is usually visible at the boundary of grain. While, in contrast, homogeneous and continuous stress field and strain field is produced on traditional macroscale in homogeneous, continuous and isotropic model, as shown in figure 4 (a), (b) and (c).

In fact, mutations of stress and strain occur in discontinuous places under the action of loading. There are some references in related experimental paper [8]. At the same time, the simulation results also conform to stress concentration phenomenon in material mechanics and Saint-Venant principle in elastic mechanics. Therefore, mutations of stress and strain inside each grain within polycrystal tend to occur at grain boundary. These places are usually the source of plasticity and fatigue, embodying the effect of intergranular interactions, which is in conformity with the phenomenon in crystal plasticity [9].

\section{Conclusion}

This paper make an effective attempt to set up polycrystalline meso-structure model based on Voronoi diagram principle in large commercial finite element software ABAQUS, combining MATLAB with Python language programming.

(1)By mesoscopic modeling, considering anisotropy and the differences of orientation of each grain in polycrystal, which have an influence on mechanical property of material under the action of loading for polycrystalline material, this research provide certain referential significance for people to have a further understanding of mesoscopic mechanism in plasticity, damage, fatigue and fracture as well as design and improvement of machining process for polycrystalline material.

(2)Compared with finite element analysis in homogeneous and isotropic model on traditional macroscale, finite element simulation in polycrystalline model on mesoscopic scale can more reasonably express the deformation process of polycrystalline material, give material performance more reasonable explanations, besides, get closer to the actual situation.

\section{References}

[1] Ziqiang Wang, Duanping Zhu. Plastic mesoscopic mechanics. Science press. Beijing. 1995.

[2] Si Liang-ying, Deng Guan-yu, etc. Polycrystal geometry modeling of crystal plasticity finite element method with voronoi diagram [J]. Journal of Materials and Metallurgy. 2009(8):193-216.

[3] Ren Huai-hui, Li Xu-dong, Li Jun-chen, Long Di-de. Mechanical response computation of two-dimensional polycrystalline material microstructure [J]. Journal of Wuhan University of Science and Technology(Natural Science Edition). 2008(31):427-431.

[4] Zhang Feng-guo, Dong Xiang-huai. Modeling of meso-structure material in microforming simulation [J]. Die and Mould Technology. 2011(3):16-19.

[5] Xiao Jian, Lin Hai-bo. Python programming basis. Tsinghua university press. Beijing. 2003.

[6] Cao Jin-feng, Wang Xu-chun, Kong Liang. Python language application in ABAQUS. Machinery industry press.

[7] Asaro RJ, Needleman A. Texture development and strain hardening in rate dependent polycrystals. Acta Metal. 1985. 33:923-953.

[8] Xiao Shui-sheng, Chen Yong-jin, Li Shao-jun. Plate and strip rolling head bending impact factor analysis based on uniform experiment [J]. Science Technology and Engineering. 2011(10). 
[9] Y. Chen, R. Guo, K. Wang, etc. The Effect of Strain Field on Applied Mechanics and Materials. Vols.217-219 (2012) pp1938-1941. 